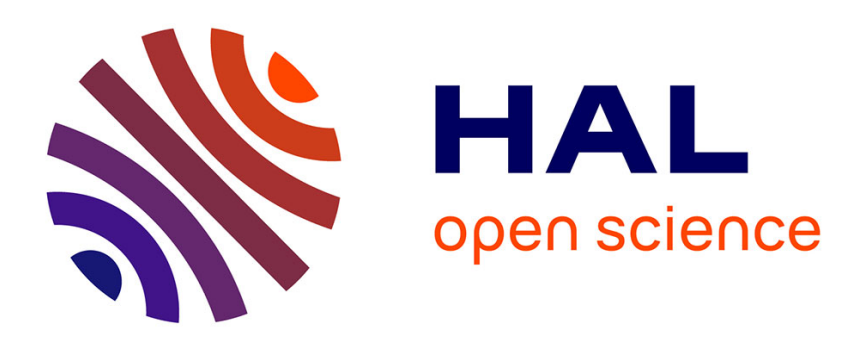

\title{
Full field modal measurement with a single standard camera
}

Clément Jailin

\section{To cite this version:}

Clément Jailin. Full field modal measurement with a single standard camera. Optics and Lasers in Engineering, 2018, 107, pp.265-272. 10.1016/j.optlaseng.2018.03.031 . hal-01762384

\section{HAL Id: hal-01762384 \\ https://hal.science/hal-01762384}

Submitted on 10 Apr 2018

HAL is a multi-disciplinary open access archive for the deposit and dissemination of scientific research documents, whether they are published or not. The documents may come from teaching and research institutions in France or abroad, or from public or private research centers.
L'archive ouverte pluridisciplinaire HAL, est destinée au dépôt et à la diffusion de documents scientifiques de niveau recherche, publiés ou non, émanant des établissements d'enseignement et de recherche français ou étrangers, des laboratoires publics ou privés. 


\title{
Full field modal measurement with a single standard
}

\section{camera}

\author{
Clément Jailin ${ }^{\mathrm{a}}$ \\ ${ }^{a}$ LMT (ENS Paris-Saclay/CNRS/Univ. Paris-Saclay) \\ 61 avenue du Président Wilson, F-94235 Cachan (FRANCE)
}

\begin{abstract}
The full field measurement of 8 vibration modes of a cantilever plate, up to the $\mathrm{kHz}$ frequency is performed using a single standard camera. The image acquisition is carried out at low rate but using fast exposure time $(1 / 4000 \mathrm{~s})$ that "freezes" a part of the motion. A deflectometry setup coupled with digital image correlation analysis allows angle fields, then deflection fields, to be measured. An out of plane displacement database is generated from all stored fields. This snapshot library is finally post-processed using principal component analysis techniques and adapted weights that enable space vibration fields to be extracted. A test case is performed on a reflecting cantilever steel plate. 370 deflection fields are measured allowing 8 space vibration fields to be extracted. The natural frequencies of those modes are estimated using the Rayleigh ratio. A coarse and visual comparison with a numerical simulation shows similar results for 6 of the 8 space modes.

Keywords: Full field measurement, Deflectometry, DIC, Modal analysis, Principal Component Analysis
\end{abstract}

\section{Introduction}

The analysis of the displacement fields of a sample during a mechanical test is a key element for model validation and identification. One of the developed full field measurement procedure is Digital Image Correlation (DIC) [1] that provides 
2D displacement fields from a series of images. This technique is now widely used in laboratories because of its robustness, its accuracy and the generally accessible required experimental setup. Based on reduced predefined kinematic solutions (e.g., finite element mesh kinematic with global DIC [2]), this method has been applied for complex measurements and identifications (e.g., measurement on images with poor texture, with gray level and blur changes [3], localized motions as crack initiation and propagation [4] or shear bands etc. ). It provides accurate displacement fields, up to centi-pixel, with well characterized uncertainties [5]. Nevertheless, DIC is generally limited to the analysis of in plane displacement fields. To circumvent this limitation, an extension of DIC for the measurements of 3D surface motions called stereo-DIC has been developed.

In stereo-DIC methods, the different views of the same surface enable, in addition to the in-plane motion, the out of plane displacement to be measured. Stereo-DIC has been applied in many fields including kinematic identification, material characterization [6], surface topology measurement [7, 8] etc. StereoDIC also appears to be an efficient tool for the analysis of non-flat structures with complex 3D shapes. Different experimental setups have been studied in the literature, generally combining multiple cameras, but also with a stereo-setup based on a single camera with multiple mirrors (mirror based single-camera stereo-DIC methods [9, 10]) or a prism [11] in order to have multiple views of the same characterized surface. Combined with fast cameras, stereo-DIC has been developed for the measurement of vibration displacement fields [12, 13, 14] (often applied for the study of the deflection of beams or plates). Nevertheless, those stereo-approaches may require a setup composed of multiple fast cameras [15] and a complex procedure for the calibration and DIC measurement. The analysis is also limited to the first vibration modes until the deflection become too small (particularly with fast cameras that often have low spatial resolutions). 
Deflectometry is an experimental method that allows small out of plane displacements to be assessed [16, 17, 18]. A grid or fringe pattern is imaged through a reflective sample by a camera. The deflection of the sample deforms the acquired grid patterns that are analyzed from phase measurements. This method is a gradient measurement technique that provides a full field slope identification of the surface. The height can then be obtained after integration. This method has been developed for different applications such as topography measurements [19, 20, 21], dynamic measurements for vibration, damage detection [22], mechanical identification [23, 24].

A similar method to deflectometry has been developed in the field of fluid mechanics in the past few years [25, 26]. An angle field is measured from the refraction of water instead of mirror samples. The bottom face of a water tank is covered by speckle patterns. A camera is located outside, at the top of the reservoir and images this bottom face throughout the water. A change of the fluid height (generated by wave propagation) distorts the acquired images and hence can be read as surface angle using digital image correlation and geometrical relations. The surface heights can thus be obtained from the integration of the measured angle fields. The method has also been used for transparent film motion analysis [27].

Many models and data reduction techniques [28, 29] (e.g., Principle Component Analysis (PCA), Singular Value Decomposition (svd), Karhunen-Loeve decomposition) provide modal decomposition of a problem with separated variables. The analysis of space-time fields allows modes, product of separated space and time functions, to be generated. This may constitute when truncated up to a certain number of modes a reduced basis. The relationship between vibration modes and PCA decomposition [29, 30] has been studied. It has been shown that, considering a light damping system composed of an identity mass matrix (that 
can be easily obtained from a change of variable), the space eigenmodes of the PCA decomposition could be confused with vibration modes [31, 32]. For an accurate modal measurement, the number of snapshots used for the decomposition has to be large [33].

It is proposed in this article a modal measurement of a vibrating plate using a deflectometry setup and a single standard camera. A series of speckled images are acquired at a slow rate and fast exposure time. The global DIC post-process gives a series of slope measurement snapshots with a very high sensitivity to the deflection. The slope fields are then integrated in height fields and stored in a database. Then a modal basis composed of the space modes associated to natural frequencies can be obtained using PCA decomposition techniques with a particular attention on the experimental uncertainties. An application on a vibrating cantilever plate is carried out. The 420 acquired images with a standard digital camera allows 8 vibration modes (space and time) to be identified up to $1 \mathrm{kHz}$ frequency. A comparison with the 8 first modes from a finite element simulation gives 6 similar space shapes.

\section{Method}

\subsection{Deflectometry}

The out of plane measurement method used in this article is based on deflectometry [16, 17, 18]. Instead of measuring the displacement field on the surface of a sample, as it would be done in DIC, it is proposed to work on the reflection of a fixed speckled image by a mirror specimen. With the deflection of the sample, the reflected image captured by the camera is distorted. Contrary to standard full field measurement methods as DIC, the deflectometry fields are slope fields that can be integrated in one single height field. For the experimental measurement 
on non-reflecting materials, a thin mirror film could be added on the surface. A drawing of the deflectometry setup is shown figure 1

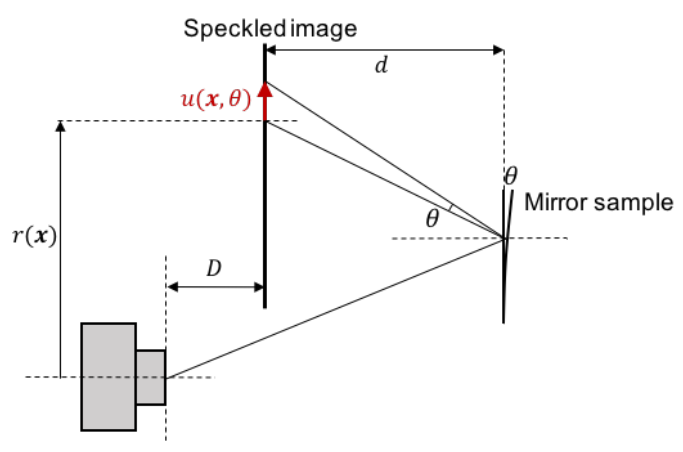

Figure 1: Principle of deflectometry based on a mirror sample that reflects a speckled image. The bending of the measured plate deforms the acquired images.

A very high out of plane sensitivity is expected, related to the geometrical parameters (i.e., length from the mirror to the speckled image). It can be noted that this length is easily adaptable depending on the required angle amplification and allowing to avoid caustic effects (the size of the speckles can also be adjusted).

Multiple sources of image distortion may appear in this procedure and can be corrected

- distortion of the image due to the deformation of the mirror sample. This is the identified distortion, related to the bending of the imaged surface. The slope fields have then to be post-processed in order to obtain the deflection.

- distortion introduced by the imaging device and setup (tilted position of the camera, conic angle of view etc. ). This can be measured and corrected for example with geometric models or with the comparison of a known speckled target (calibration printed sample) with its distorted acquisition.

- distortion introduced by an initial curvature on the reflecting sample. This can be identified using a calibration sample. 
The two last distortions can be identified experimentally or analytically (with assumptions on the geometry). Because the following application is the measurement of an assumed plane sample (a steel cantilever plate), the last distortion is not considered.

\subsection{Digital image correlation}

Digital image correlation [1] relies on the registration of an image $f(\boldsymbol{x})$, defined for every pixels of the selected region of interest $\boldsymbol{x}=[x, y]$ in the reference configuration and a series of pictures $g(\boldsymbol{x})$ in the deformed configurations. The registration operation consists of minimizing the sum of squared differences between the deformed image corrected for its displacement and the reference image. Hence

$$
\boldsymbol{u}(\boldsymbol{x})=\operatorname{Argmin}_{u \in E} \frac{1}{2 \sigma^{2} N_{x}} \sum_{\boldsymbol{x}}(f(\boldsymbol{x})-g(\boldsymbol{x}+\boldsymbol{u}(\boldsymbol{x})))^{2}
$$

with $E$, a subspace composed of a reduced kinematic basis, $\sigma$ the standard deviation of the supposed white Gaussian noise and $N_{x}$ the number of pixels per image. With global DIC [2], the motion is described on a finite element mesh kinematic basis using standard shape functions $\boldsymbol{\phi}_{i}(\boldsymbol{x})$ and a reduced number of unknowns $u_{i}$ such that

$$
\boldsymbol{u}(\boldsymbol{x})=\sum_{i=1}^{N_{\mathrm{dof}}} u_{i} \boldsymbol{\phi}_{i}(\boldsymbol{x})
$$

with $N_{\text {dof }}$ the total number of degrees of freedom (2 displacements per node). The minimization of the functional is carried out with a Newton descent algorithm. The DIC is carried out using the Correli-3.0 framework [34] with an update at

each iteration $l$ of the displacement field $\{\boldsymbol{u}\}^{l}=\{\boldsymbol{u}\}^{l-1}+\{\delta \boldsymbol{u}\}$. The correction of the displacement $\{\delta \boldsymbol{u}\}$ for the linearized problem can be written

$$
\{\delta \boldsymbol{u}\}=[\boldsymbol{H}]^{-1}\{\boldsymbol{b}\}
$$


with $[\boldsymbol{H}]$ the Hessian function with respect to $\{\boldsymbol{u}\}$ and $\{\boldsymbol{b}\}$ the second member vector

$$
\begin{gathered}
H_{i j}=\frac{1}{2 \sigma^{2}} \sum_{\boldsymbol{x}}\left(\nabla f(\boldsymbol{x}) \boldsymbol{\phi}_{i}(\boldsymbol{x})\right)\left(\boldsymbol{\phi}_{j}(\boldsymbol{x}) \nabla f(\boldsymbol{x})\right) \\
b_{j}=\frac{1}{2 \sigma^{2}} \sum_{\boldsymbol{x}} \eta^{l}(\boldsymbol{x}) \boldsymbol{\phi}_{j}(\boldsymbol{x}) \nabla f(\boldsymbol{x})
\end{gathered}
$$

with $\nabla$ the gradient operator and $\eta^{l}(\boldsymbol{x})$ the residual field at iteration $l$ that represents what has not been captured by the kinematic correction. This residual allows the procedure to be (in)validated

$$
\eta^{l}(\boldsymbol{x})=f(\boldsymbol{x})-g\left(\boldsymbol{x}+\boldsymbol{u}^{l}(\boldsymbol{x})\right)
$$

From geometrical or experimental relations, the measured displacement fields on the deformed surface can be read as angle fields. With $\{\boldsymbol{\theta}\}=\left[\theta_{x}, \theta_{y}\right]^{t}$ the angles in $x$ and $y$ directions

$$
\{\boldsymbol{\theta}\}=\Gamma(\{\boldsymbol{u}\})
$$

This function will be expressed for the test case in the application part considering a simple cone field of view correction.

\subsection{Integration of the measured field}

The measured bending angles of the plate $\theta_{x}(\boldsymbol{x})$ and $\theta_{y}(\boldsymbol{x})$ can be integrated into the out of plane displacement of the plate $\{\boldsymbol{h}\}$. The bending angles can easily be related to the height variations (deflection of the plate)

$$
\left[\theta_{x}, \theta_{y}\right]^{t}=\left[\frac{\partial h}{\partial y}, \frac{\partial h}{\partial x}\right]^{t}
$$

The two terms are written in matrix notation

$$
\{\boldsymbol{\theta}\}=[\boldsymbol{S}]\{\boldsymbol{h}\}
$$


where $[\boldsymbol{S}]$ of size $\left[N_{\text {dof }}, N_{\text {dof }} / 2\right]$ defines the linear operation on $\{\boldsymbol{h}\}$ to produce the gradient of $\{\boldsymbol{h}\}$ in $y$ and $x$ direction. As proposed in [25], the integration of the two previous equations is performed through a linear least square minimization

$$
\{\boldsymbol{h}\}=\operatorname{Argmin}_{h}\|[\boldsymbol{S}]\{\boldsymbol{h}\}-\{\boldsymbol{\theta}\}\|_{\boldsymbol{H}}^{2}
$$

where $\|\cdot\|_{\boldsymbol{H}}$ denotes the $[\boldsymbol{H}]$ norm thus weighted by the correlation matrix of the DIC procedure [35, 36]. The result is hence weighted by the measurement uncertainty. The linear system that has to be solved is

$$
\{\boldsymbol{h}\}=\left[\boldsymbol{H}_{h}\right]^{-1}[\boldsymbol{S}]^{t}[\boldsymbol{H}]\{\boldsymbol{\theta}\}
$$

with $\left[\boldsymbol{H}_{h}\right]=[\boldsymbol{S}]^{t}[\boldsymbol{H}][\boldsymbol{S}]$ the Hessian matrix that corresponds to integration uncertainty.

It can be noticed that the integrated displacement field is composed of an unknown constant. Its value can be obtained with additional out of plane measurement points (at least one) such as a LVDT (if the constant motion is expected to be in the sensor acquisition frequency range), accelerometer behind the surface, an orthogonal camera etc. or with assumptions on the displacement field. In our application, the mean displacement at the fixed boundary condition of the plate has been globally subtracted.

\subsection{Modal measurement}

Space measurements: The previous analysis and integration enables the measurement of the deflection of the plate for each acquired time step. These out of plane displacement fields are then stored and can be post-processed in order to obtain the main spatial modes. Form [31, 33, 32], it has been shown that the PCA converges to linear normal modes in multi-modal free responses of symmetric linear systems, but only if the mass matrix $[\boldsymbol{M}]$ is proportional to the 
identity matrix (which can be achieved by a coordinate transformation if the mass distribution is known) and when the system is lightly damped. The equation of an unforced, undamped linear multi-degree-of-freedom vibration system, with an identity equivalent mass matrix is

$$
\frac{\partial^{2}\{\tilde{\boldsymbol{h}}\}}{\partial t^{2}}+[\boldsymbol{M}]^{-1 / 2}[\boldsymbol{K}][\boldsymbol{M}]^{-1 / 2}\{\tilde{\boldsymbol{h}}\}=0
$$

with the displacement field $\{\boldsymbol{h}\}=[\boldsymbol{M}]^{-1 / 2}\{\tilde{\boldsymbol{h}}\}$ and $[\boldsymbol{K}]$ the stiffness matrix, defined (as for the mass matrix) using the same global-DIC finite element mesh.

A large set of images is required to correctly catch the first modes. In order to take into account the measurement and the integration uncertainty, the PCA decomposition can be weighted by the square root of the Hessian function $\left[\boldsymbol{H}_{h}\right]$ (as proposed in [37]). Then the weighted displacement fields $\{\boldsymbol{h}(\boldsymbol{x}, t)\}$ composed of few different loading steps (indexed by $t$, a dimensionless number) are expressed as the sum over $N_{n}$ modes, product of two functions with separate variables, one is a "temporal" evolution $\left\{\boldsymbol{\tau}^{n}(t)\right\}$ (that is not considered in the following) and space function $\left\{\boldsymbol{q}^{n}(\boldsymbol{x})\right\}$ such that

$$
\left[\boldsymbol{H}_{h}\right]^{1 / 2}[\boldsymbol{M}]^{1 / 2}\{\boldsymbol{h}(\boldsymbol{x}, t)\}=\sum_{n=1}^{N_{n}}\left[\boldsymbol{H}_{h}\right]^{1 / 2}[\boldsymbol{M}]^{1 / 2}\left\{\boldsymbol{\tau}^{n}(t)\right\}\left\{\boldsymbol{q}^{n}(\boldsymbol{x})\right\}
$$

The amplitudes of the mode may be applied on $\left\{\boldsymbol{\tau}^{n}(t)\right\}$ or $\left\{\boldsymbol{q}^{n}(\boldsymbol{x})\right\}$ (it has been chosen in the following a norm for $\left\{\boldsymbol{\tau}^{n}(t)\right\}$ of 1 hence the norm of $\left\{\boldsymbol{q}^{n}(\boldsymbol{x})\right\}$ is the mode amplitude).

Instead of working with all the $N_{n}$ modes, it is possible to truncate the sum and generate a reduced basis composed of few main modes (with the highest eigenvalues). Looking at the residual field (i.e., the sum of all removed modes) allows the truncation to be (un)validated if it has low values and does not contain space correlation (i.e., would be considered as a white noise). 
Temporal evolution: In this procedure, it is proposed to capture snapshots of the vibrating plate. The images are acquired few order of magnitudes slower than the mode frequency and at random time. Hence the temporal evolution of the PCA decomposition will not be considered. Because the PCA analysis deals with a L2 norm, weighting the displacements by the square root of the mass matrix corresponds to an energetic norm. The resulting eigenmodes are also orthogonal with respect to the system mass.

Another approach is to compute the natural frequencies from the Rayleigh ratio. With known spatial modes of the plate and both mass and stiffness matrix (computed on the same finite element mesh as the one used in the global DIC), it is possible to compute the natural frequency of each mode. The Rayleigh-Ritz ratio (based on the equality between the kinematic and potential energy) can be expressed considering the eigenvectors $\left\{\boldsymbol{q}^{n}\right\}$

$$
\left(\omega^{n}\right)^{2}=\frac{\left\{\boldsymbol{q}^{n}\right\}^{t}[\boldsymbol{K}]\left\{\boldsymbol{q}^{n}\right\}}{\left\{\boldsymbol{q}^{n}\right\}^{t}[\boldsymbol{M}]\left\{\boldsymbol{q}^{n}\right\}}
$$

The more PCA mode studied, the more the spatial function is expected to be composed of high noise frequency patterns. The Rayleigh ratio is highly sensitive to this noise that pollutes the natural frequency estimation. Smoothing constraints could be added using Integrated-DIC [35] approaches where the solution is directly expressed on a highly reduced kinematic basis, generated from a vibration model. Local elastic regularizations [2] would also give lower frequency fields depending on the chosen regularization length.

The entire procedure, from image acquisition to modal analysis can be summarized by the 5 successive steps shown in table 1 . 


\begin{tabular}{lc}
\hline Step & Outputs \\
\hline Acquisition of all images & $f(\boldsymbol{x})$ and $g(\boldsymbol{x}, t)$ \\
\hline DIC measurement & $\{\boldsymbol{\theta}(\boldsymbol{x}, t)\}$ and $[\boldsymbol{H}]$ \\
\hline Integration of the angle & $\{\boldsymbol{h}(\boldsymbol{x}, t)\}$ and $\left[\boldsymbol{H}_{h}\right]$ \\
\hline Space mode analysis & $\left\{\boldsymbol{q}(\boldsymbol{x})^{n}\right\}$ \\
\hline Natural frequency computation & $\omega^{n}$ \\
\hline
\end{tabular}

Table 1: Summarized successive steps of the entire procedure with the output quantities

\section{Application to the modal analysis of a plate}

\subsection{Test case presentation}

The test case is the vibration of a rectangular stainless steel plate $\left(145 \times 85 \times 1.0 \mathrm{~mm}^{3}\right)$, fixed at one of the 4 edges, excited in the back side with a hammer at different and random space positions. The surface of the plate has been polished to reflects a printed speckled image, located between the camera and the mirror. The distance between the sample and the speckled image is $d=26.0 \mathrm{~cm}$ and the distance between the speckled image and the imaging device is $D=48.0 \mathrm{~cm}$. A picture of the experimental setup is presented figure 2, The elastic mechanical properties for the stiffness matrix are the Young modulus $E=205 \mathrm{GPa}$ and the Poisson ratio $\nu=0.27$ and the density for the mass matrix is $\rho=8050 \mathrm{~kg} \cdot \mathrm{m}^{-3}$.

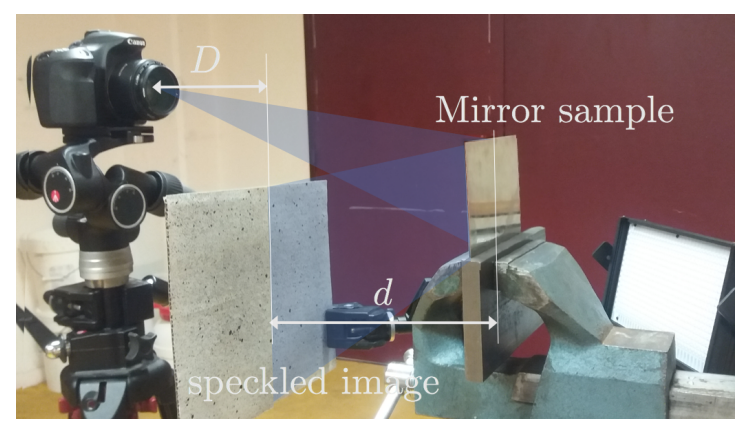

Figure 2: Experimental deflectometry setup for the modal analysis of a plate 
The camera is a Canon 60D with a $50 \mathrm{~mm}$ objective, exposure time $1 / 4000 \mathrm{~s}$, f-10 with a LED panel for a diffuse light. 420 images are taken at random times, for different hammer shock positions (acquired few seconds after the shock to avoid transitory phenomenon). The resolution, measured (i) on the speckled image is 1 pixel $\rightarrow 218 \mu \mathrm{m}$ and (ii) on the mirror sample is 1 pixel $\rightarrow 161 \mu \mathrm{m}$. Because the images are taken with a detector parallel to the sample and speckled image, the Region Of Interest (ROI) is located in the bottom part (see figure 22. The top part of the acquired image is thus composed of the black background and is cropped. It is noteworthy that the camera could also be tilted with an angle in order to capture the mirror image with a larger detector zone. A geometric correction of this angle distortion would have to be performed.

The acquisition frequency is approximately $1-2 \mathrm{~Hz}$ thus is not fast enough to capture the vibration of the structure (first mode at approximately $123 \mathrm{~Hz}$ ). Nevertheless, the exposure time is chosen so that the images are "frozen" and not blurred by the vibration of the searched modes $(1 / 4000 \mathrm{~s}$ was enough and is a standard available setting for most of the commercial digital camera otherwise 1/8000 s would have also been selected with a higher luminosity or ISO sensitivity).

One of the acquired image is shown figure 3 (a) with 3 free edges and one fixed boundary condition at the bottom edge by a clamping device (that finally was found from the following results to not be perfect).

\subsection{Radial distortion correction}

Considering the flat mirror sample, the distortion by the camera can be evaluated and corrected analytically. The measured point, figure [1, is defined at a position $r^{2}=x^{2}+y^{2}$ from the vertical projection of the camera. Hence the deflection angle can be related to the identified displacement field (identified using the resolution at the speckled image). The angle fields can be written 


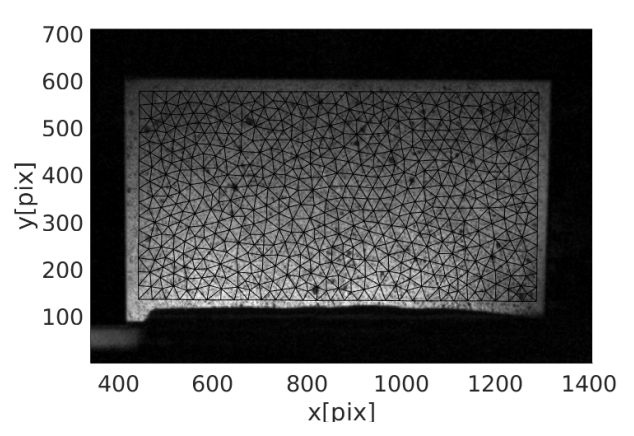

Figure 3: Reference of the reflected speckled image $f(\boldsymbol{x})$ with the selected mesh on the ROI. Note that the bottom boundary condition does not look straight but is in fact the reflect of the front grip thickness.

$$
\begin{aligned}
& \boldsymbol{\theta}(\boldsymbol{x})=\Gamma[\{\boldsymbol{u}(\boldsymbol{x})\}]=\tan ^{-1}[\boldsymbol{u}(\boldsymbol{x}) / F(\boldsymbol{x})] \text { with } \\
& \qquad F(r)=2 d\left(1+\left(\frac{r}{D+2 d}\right)^{2}\right)
\end{aligned}
$$

It is noteworthy that this cone distortion correction is quite small (because the solid angle is small).

\subsection{Dynamic deflection measurements}

A static validation of the procedure has been performed and is shown in an appendix. The DIC procedure is applied in the ROI of the acquired images, corresponding with the black mesh added on figure 3. Only a small band around the $\mathrm{ROI}$ is not considered in order to remove the correlation on the edges that may disappear because of convex bending. The T3 (3 nodes elements with bi-linear shape functions) element mesh size used for the global DIC procedure is approximately 30 pixels (for a total of 611 elements). The procedure does not converge for 50 images because of high displacements and blur on the image. Finally, 370 displacements fields are measured. 30 of these fields have been acquired without vibration of the plate, in the reference configuration, in order to quantify the 
uncertainty. The standard deviation of the measured displacements on those 30 reference fields is 0.032 pixel.

Long wave spatial patterns are expected for the first modes hence a finner DIC mesh is not required. The two angle fields $\theta_{x}(\boldsymbol{x})$ and $\theta_{y}(\boldsymbol{x})$ at convergence are shown figure $4(\mathrm{a}-\mathrm{b})$.

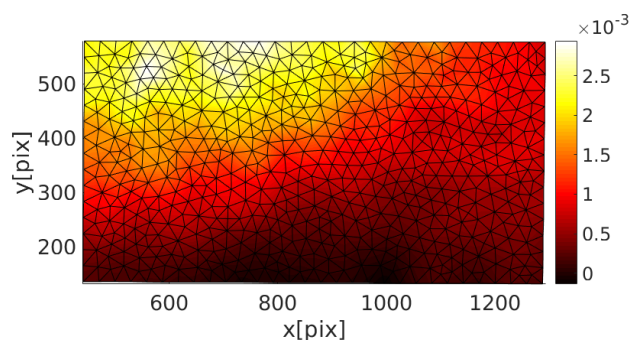

(a) $\theta_{x}(\boldsymbol{x})$

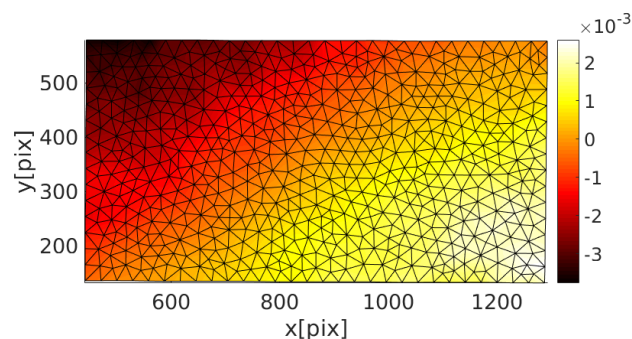

(b) $\theta_{y}(\boldsymbol{x})$

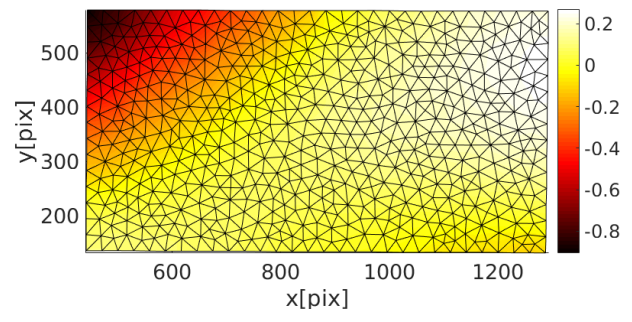

(c) $h(\boldsymbol{x})$

Figure 4: Measured deflection angle fields, at step 98 for (a) $\theta_{x}(\boldsymbol{x})$ (b) $\theta_{y}(\boldsymbol{x})$. The two fields are expressed in radian and (c) the integrated field corresponding to the height, expressed in pixels. The amplitude of the deflection is less than one pixel ( 1 pixel measured on the sample $\leftrightarrow 161 \mu \mathrm{m})$. Note that this field is defined by an unknown constant.

The mean norm of the final residual fields decreases from $9.4 \%$ of the gray level dynamic to $2.1 \%$ that corresponds to the residual value of the 30 first unloaded images $(1.9 \%))$. No localized patterns are visible on the residual fields. The captured kinematic and the DIC procedure can thus be validated. These angular fields can be numerically integrated using the weighted least square method. The 
result is a single out of plane field per state. The deflection of the plate, in pixel (i.e., resolution of the mirror sample), is shown for step 98 in figure 4(c). It can be noted that because this field is the result of an integration, it is not very sensitive to the noise. Two other fields of the database that are clearly different from the field time 98 are presented figure 5 .
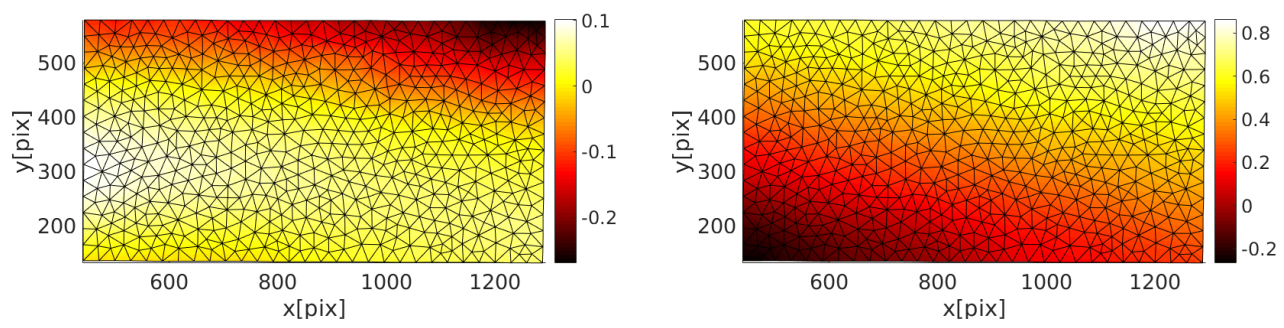

Figure 5: Two other deflections of the plate $h(\boldsymbol{x}, t)$ at step $t=30$ and $t=65$, expressed in pixel. All deflections fields constitute a deflection database then post-processed to extract the principal components.

The same DIC analysis is hence carried out for the 420 images that provides 370 converged fields. Those fields are stored in a library that has to be postprocessed to extract the principal space fields related to vibration modes.

\section{Modal measurement}

\subsection{Space modes}

From the stored out-of-plane displacement fields of the 370 time steps, vibration modes can be extracted. The PCA decomposition of the data gives the first space modes of the plate. As expected by the standard elasticity, the more modes the higher the spatial frequency. Finally 8 modes can be identified easily until the function became too much affected by high frequency spatial patterns (see figure 6). The 9th mode does not contain low spatial coherency but is composed of noise. 


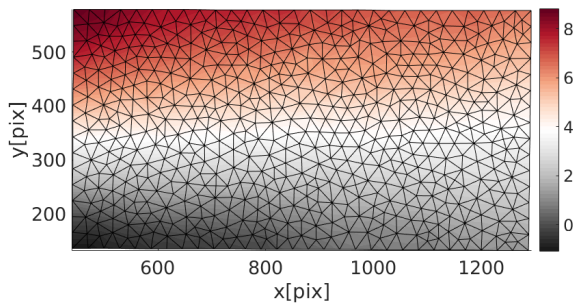

(1) $\boldsymbol{q}(\boldsymbol{x})^{1}$

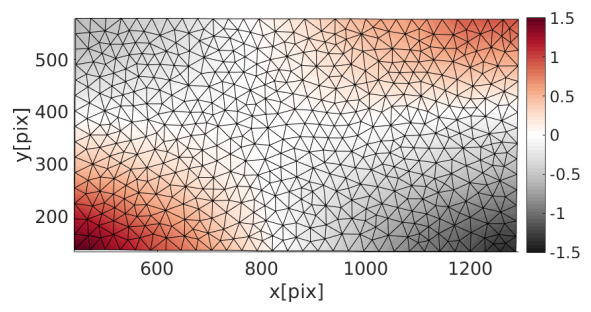

(3) $\boldsymbol{q}(\boldsymbol{x})^{3}$

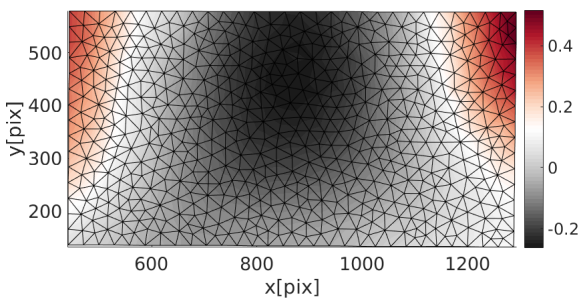

(5) $\boldsymbol{q}(\boldsymbol{x})^{5}$

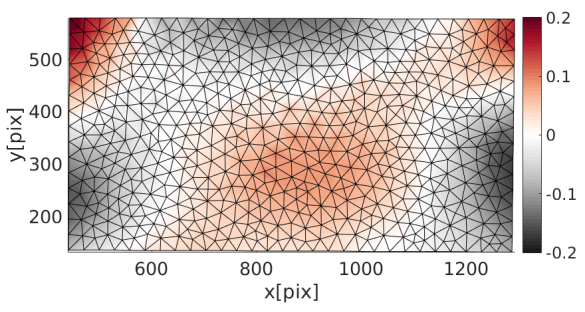

(7) $\boldsymbol{q}(\boldsymbol{x})^{7}$

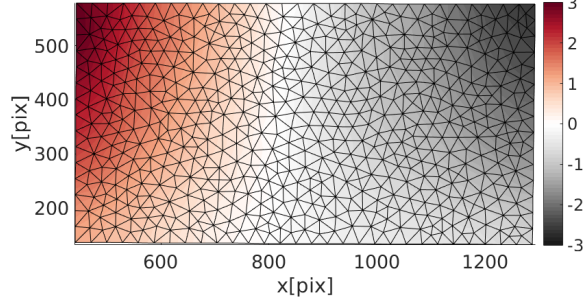

(2) $\boldsymbol{q}(\boldsymbol{x})^{2}$

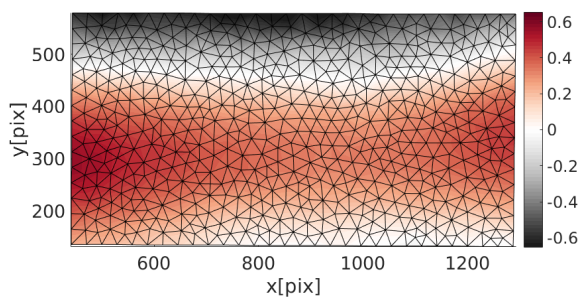

(4) $\boldsymbol{q}(\boldsymbol{x})^{4}$

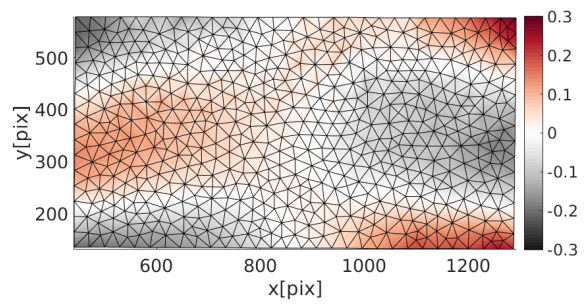

(6) $\boldsymbol{q}(\boldsymbol{x})^{6}$

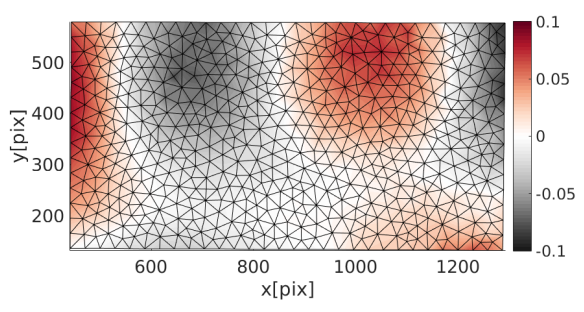

(8) $\boldsymbol{q}(\boldsymbol{x})^{8}$

Figure 6: The eight first experimental space fields with the highest eigenvalues $\boldsymbol{q}(\boldsymbol{x})^{n}$. A symmetric divergent color map has been used to highlight the positive and negative values (except for mode 1 and 5). 
The analysis of modes 3 and 6 shows that the applied boundary conditions are not a corresponding to a perfect cantilever plate but some displacement fields are seen in the bottom edge of the ROI. Although the clamping device was tight, the shapes of the two grips were slightly curved leading to boundary conditions that were not applied uniformly.

It can be noticed that the last identified modes are composed, in addition to the expected wave shape, of high frequency patterns (with a length much smaller than the expected deformation shape) that will pollute the Rayleigh ratio. Using regularized approaches or applying a filter would allow the different frequencies to be separated.

A simple elastic finite element simulation in order to extract the first numerical modes enables a coarse and visual experimental/numerical comparison to be carried out. The simulation is performed on a slightly larger mesh in order to model the entire plate and not only the ROI (it corresponds to a larger rectangle 25 pixels outside the three free edges and 50 on the fixed edge). The field is then plotted on the DIC mesh for a better comparison. The first identified modes have a natural frequency of respectively $[123,213,443,772,859,935,1256,1586] \mathrm{Hz}$. The numerical results are shown figure 7 .

On the eight presented modes, 6 can be directly compared, namely the numerical modes $[1-2-3-4-5-7]$ to the experimental modes $[1-2-5-4-8-7]$. The last modes are measured with an out of plane displacement amplitude of 0.1 pixel $(29 \mu \mathrm{m})$ amplitude that highlights the huge sensitivity of the method (note that this amplitude is related to the temporal function amplitude whose L2 norm is 1$)$. 


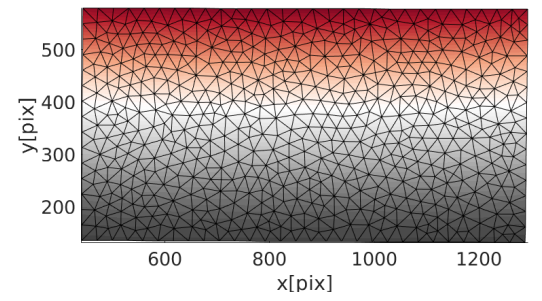

(1)

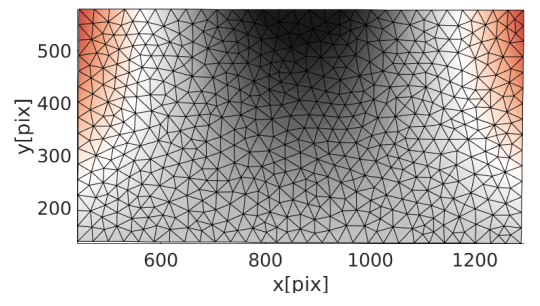

(3)

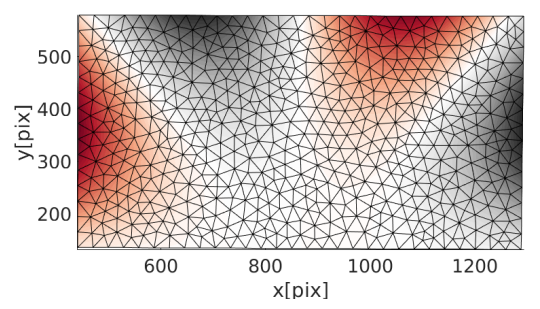

(5)

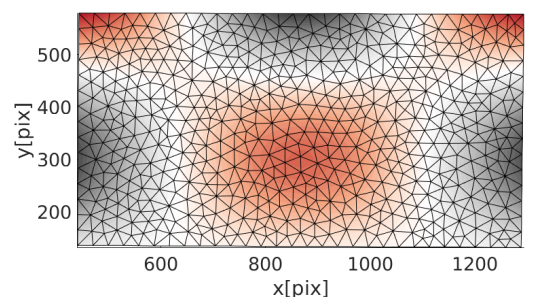

$(7)$

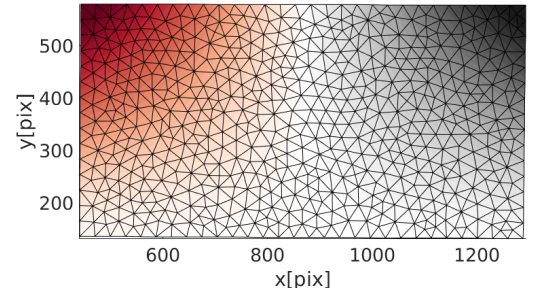

$(2)$

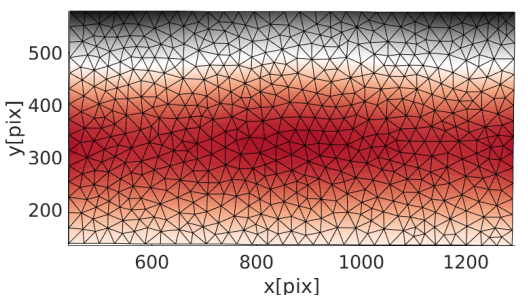

$(4)$

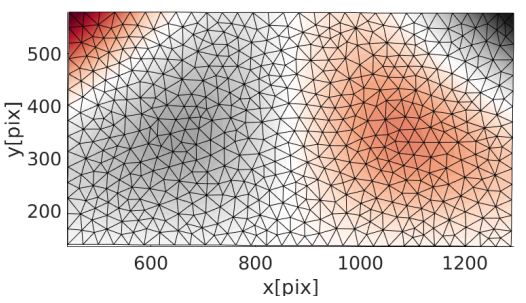

(6)

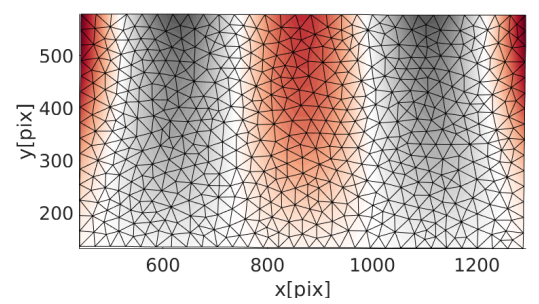

(8)

Figure 7: The eight first numerical space fields. The same color maps dynamic as the experimental results are used. 


\subsection{Temporal modes}

With the identified spatial modes, the Rayleigh ratio allows the natural frequency to be obtained from the mass and stiffness matrices. Table 2 shows the frequencies associated to each modes and their comparison with the equivalent numerical modes.

Table 2: Computed natural frequency using Rayleigh ratio compared with the 6 corresponding numerical modes.

\begin{tabular}{|c|c|c|c|c|c|c|c|c|}
\hline Experimental PCA mode & 1 & 2 & 3 & 4 & 5 & 6 & 7 & 8 \\
\hline Experimental frequency [Hz] & 113 & 241 & 456 & 666 & 476 & 2945 & 2496 & 1672 \\
\hline Corresponding numerical mode & 1 & 2 & - & 4 & 3 & - & 7 & 5 \\
\hline Numerical frequency [Hz] & 123 & 213 & - & 772 & 442 & - & 1256 & 859 \\
\hline
\end{tabular}

There is a good correlation between the natural frequencies of the experimental modes $1-2-4-5$ and the equivalent shapes of the finite element model for modes $1-2-4-3$. The natural frequency of the experimental mode 4 is higher than mode 5 as expected from the simulation (also for mode 7 and 8 even if the accuracy of the value can be discussed).

As previously discussed, the last modes (after mode 5) are polluted by high frequency patterns that affect the natural frequency computation from the Rayleigh ratio. A more regularized approach would consist of identifying a smooth model (composed of long wave functions for example) and find the natural frequencies of this model.

Experimental mode 7 clearly corresponds to an equivalent numerical mode (number 7) of $1255 \mathrm{~Hz}$. For a standard full field measurement of this mode, a stereo camera setup with a acquisition frequency of at least 5 to $10 \mathrm{kHz}$ would have been required. 


\section{Conclusion and discussion}

The measurement of the 8 first modes of a vibrating cantilever plate has been performed using a deflectometry setup composed of a single standard camera. Set with a fast exposure time $(1 / 4000 \mathrm{~s})$, a Canon $60 \mathrm{D}$ camera acquires a printed speckled image through a vibrating stainless steel mirror sample. This fast exposure time "freezes" the apparent deformation. A global digital image correlation procedure allows this apparent deformation of the plate to be measured and related to the curvature of the sample. Then the height fields are computed from the integration of these angle fields and stored for all acquisition steps. This database is finally weighted by the adapted measurement uncertainties and mass matrix to be post processed in order to extract the principal components that correspond to the space vibration fields. From the Rayleigh ratio, the natural frequencies of all these experimental modes can be estimated. Modes with a very small amplitudes and frequencies higher than $1 \mathrm{kHz}$ are identified. With standard stereo-DIC approaches, this would have required fast stereo cameras much more expensive and arduous to carry out.

A comparison with the numerical modes obtained from a finite element simulation highlights the relevance of the measured modes even if the boundary conditions may not have been perfectly modeled. Similar space results can be noted for

6 modes. The natural frequencies of those 6 equivalent modes are corresponding for 4 of them.

The space results show that the fixed boundary conditions are not perfect and some out of plane displacements are visible. Because this boundary conditions have been modeled as perfect in the finite element simulation, two experimental modes (number 3 and 6) cannot be compared with the numerical simulation.

The accuracy of the PCA method is based on a large database (i.e., a large 
number of fields). In the presented application, the same analysis performed on 75 images gives similar results. Nevertheless, the reason why the eigenvalue positions of two space modes were inverted compared with the numerical results and the Rayleigh ratio may be related to the image sampling that was not important enough , wrong model of the boundary conditions or the excitation that was not really random.

It can be seen that the last identified modes are polluted by high spatial patterns considered as noise. This noise affects the Rayleigh ratio. This problem could be circumvented by adding regularization with predefined fields or a local elastic constraint with an adequate regularization length.

To go further than a visual comparison between the experimental results and the numerical simulation, an identification procedure could be performed. The mechanical parameters as well as the real boundary conditions could be identified using for example Finite Element Method Updating or Integrated-DIC [35].

\section{Acknowledgements}

The author would like to acknowledge particularly Stéphane Roux for useful discussions on the PCA decomposition, Thomas Jailin for his valuable help on the simulation part and Manon Debayle for a careful proofreading of the manuscript.

\section{Appendix: Static validation}

A static validation of the procedure on the same sample is performed. The sample is loaded with an out of plane force at the top left corner. 10 reference images and 3 steps under small loads are acquired: $0.31,0.40$ and $0.50 \mathrm{~N}$. The distance between the sample and the speckled image is $d=50.2 \mathrm{~cm}$ and the

distance between the speckled image and the imaging device is $D=41.0 \mathrm{~cm}$. The 
same imaging device is used. The resolution measured (i) at the speckled pattern is $287 \mu \mathrm{m}$ and (ii) at the mirror sample $197 \mu \mathrm{m}$.

The T3 element mesh size used for the global DIC procedure is approximately 50 pixels (for a total of 287 elements). A finite element simulation of the bending, with fixed boundary conditions is performed on a fine mesh and projected on the DIC mesh (i.e., smaller than the real plate as in figure 3).

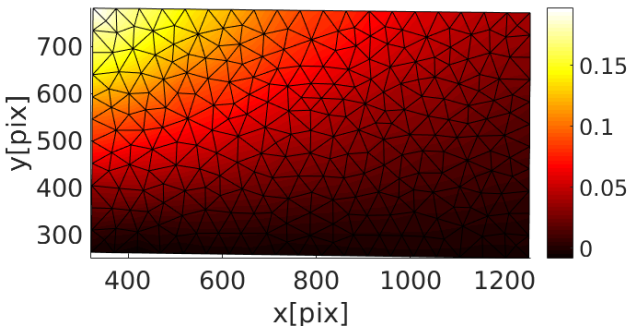

(1)

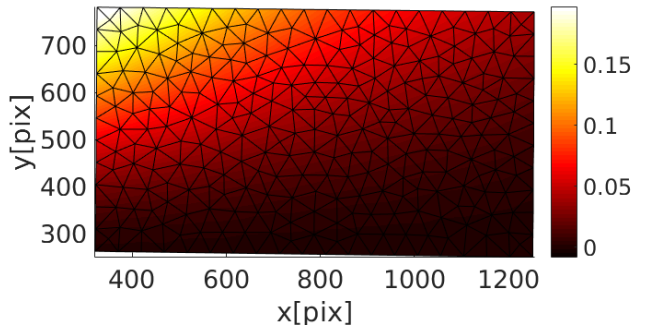

$(2)$

Figure 8: Deflection fields for the load at $0.50 \mathrm{~N}$, expressed in pixels (1 pixel $\leftrightarrow 197 \mu \mathrm{m})$ for the static test case for (1) the measured field with the proposed method and (2) the simulated field

A comparison between experiment and numeric is presented figure 8 for the third loading step $(0.50 \mathrm{~N})$. A good agreement between the two fields can be noted. For the two other steps, the deflection field has the same shape but the amplitudes are slightly different between the measured and simulated fields. A mean variation of $9 \%$ and $4 \%$ for the first and second step can be noted. Those variations may come from uncertainties of the load measurement $( \pm 0.01 N)$, wrong model of the boundary conditions, elastic parameters.

\section{References}

[1] M.A. Sutton. Computer Vision-Based, Noncontacting Deformation Measurements in Mechanics: A Generational Transformation. Appl. Mech. Rev., 65(AMR-13-1009):050802, 2013. 
[2] G. Besnard, S. Guérard, S. Roux, and F. Hild. A space-time approach in digital image correlation: Movie-DIC. Optics and Lasers in Engineering, 49(1):71-81, 2011.

[3] A. Charbal, J.-E. Dufour, A. Guery, F. Hild, S. Roux, L. Vincent, and M. Poncelet. Integrated digital image correlation considering gray level and blur variations: Application to distortion measurements of ir camera. Optics and Lasers in Engineering, 78:75-85, 2016.

[4] S. Roux, J. Réthoré, and F. Hild. Digital image correlation and fracture: an advanced technique for estimating stress intensity factors of $2 \mathrm{~d}$ and $3 \mathrm{~d}$ cracks. Journal of Physics D: Applied Physics, 42(21):214004, 2009.

[5] F. Hild and S. Roux. Comparison of local and global approaches to digital image correlation. Experimental Mechanics, 52(9):1503-1519, 2012.

[6] J.-E. Dufour, F. Hild, and S. Roux. Shape, displacement and mechanical properties from isogeometric multiview stereocorrelation. The Journal of Strain Analysis for Engineering Design, 50(7):470-487, 2015.

[7] J.-E. Dufour, S. Leclercq, J. Schneider, S. Roux, and F. Hild. 3d surface measurements with isogeometric stereocorrelation-application to complex shapes. Optics and Lasers in Engineering, 87:146-155, 2016.

[8] L. Dubreuil, J.-E. Dufour, Y. Quinsat, and F. Hild. Mesh-based shape measurements with stereocorrelation. Experimental Mechanics, 56(7):1231-1242, 2016.

[9] L. Yu and B. Pan. Single-camera high-speed stereo-digital image correlation for full-field vibration measurement. Mechanical Systems and Signal Processing, 94:374-383, 2017. 
[10] B. Pan, L. Yu, and Q.B. Zhang. Review of single-camera stereo-digital image correlation techniques for full-field $3 \mathrm{~d}$ shape and deformation measurement. Science China Technological Sciences, pages 1-19, 2017.

[11] K. Genovese, L. Casaletto, J.A. Rayas, V. Flores, and A. Martinez. Stereodigital image correlation (DIC) measurements with a single camera using a biprism. Optics and Lasers in Engineering, 51(3):278-285, 2013.

[12] C. Niezrecki, P. Avitabile, C. Warren, P. Pingle, and M. Helfrick. A review of digital image correlation applied to structura dynamics. In AIP Conference Proceedings, volume 1253, pages 219-232. AIP, 2010.

[13] T. Siebert and K. Splitthof. Vibration analysis using 3D image correlation technique. In EPJ Web of Conferences, volume 6, page 11004. EDP Sciences, 2010 .

[14] P.L. Reu, D.P. Rohe, and L.D. Jacobs. Comparison of DIC and LDV for practical vibration and modal measurements. Mechanical Systems and Signal Processing, 86:2-16, 2017.

[15] R. Huňady, M. Hagara, and F. Trebuňa. The measurement of standing wave patterns by using high-speed digital image correlation. American Journal of Mechanical Engineering, 2(7):247-251, 2014.

[16] R. Huang. High precision optical surface metrology using deflectometry. The University of Arizona, 2015.

[17] L. Huang, J. Xue, B. Gao, C. McPherson, J. Beverage, and M. Idir. Modal phase measuring deflectometry. Optics express, 24(21):24649-24664, 2016. 
[18] Y.-L. Xiao, S. Li, Q. Zhang, J. Zhong, X. Su, and Z. You. Optical fringereflection deflectometry with sparse representation. Optics and Lasers in Engineering, 2017.

[19] K.K. Szwedowicz. 3D-deflectometry: fast nanotopography measurement for the semiconductor industry. PhD thesis, Technische Universiteit Eindhoven, 2006.

[20] J. Burke, W. Li, A. Heimsath, C. von Kopylow, and R.B. Bergmann. Qualifying parabolic mirrors with deflectometry. Journal of the European Optical Society-Rapid publications, 8, 2013.

[21] J.H. Burge, P. Su, G. Butel, R. Huang, A. Maldonado, and T. Su. Measuring large mirrors using scots, the software configurable optical test system. In Proc. of SPIE Vol, volume 9151, pages 91510Z-1, 2014.

[22] C. Devivier, F. Pierron, and M.R. Wisnom. Damage detection in composite materials using deflectometry, a full-field slope measurement technique. Composites Part A: Applied Science and Manufacturing, 43(10):1650-1666, 2012 .

[23] A. Giraudeau, B. Guo, and F. Pierron. Stiffness and damping identification from full field measurements on vibrating plates. Experimental Mechanics, 46(6):777-787, 2006.

[24] C. Devivier, F. Pierron, P. Glynne-Jones, and M. Hill. Time-resolved fullfield imaging of ultrasonic lamb waves using deflectometry. Experimental Mechanics, 56(3):345-357, 2016.

[25] F. Moisy, M. Rabaud, and K. Salsac. A synthetic Schlieren method for the 
measurement of the topography of a liquid interface. Experiments in Fluids, 46(6):1021, 2009.

[26] A.P. Damiano, P.-T. Brun, D.M. Harris, C.A. Galeano-Rios, and J.W.M. Bush. Surface topography measurements of the bouncing droplet experiment. Experiments in Fluids, 57(10):163, 2016.

[27] J. Krenn, P. Scharfer, and W. Schabel. Visualization of surface deformations during thin film drying using a Digital-Image-Correlation method. Chemical Engineering and Processing: Process Intensification, 50(5):569-573, 2011.

[28] I.T. Jolliffe. Principal component analysis and factor analysis. In Principal component analysis, pages 115-128. Springer, 1986.

[29] H. Abdi and L.J. Williams. Principal component analysis. Wiley Interdisciplinary Reviews: Computational Statistics, 2(4):433-459, 2010.

[30] G. Kerschen, J.C. Golinval, A.F. Vakakis, and L.A. Bergman. The method of proper orthogonal decomposition for dynamical characterization and order reduction of mechanical systems: an overview. Nonlinear dynamics, 41(1):147$169,2005$.

[31] E. Kreuzer and O. Kust. Analysis of long torsional strings by proper orthogonal decomposition. Archive of Applied Mechanics, 67(1):68-80, 1996.

[32] B.F. Feeny and Y. Liang. Interpreting proper orthogonal modes of randomly excited vibration systems. Journal of Sound and Vibration, 265(5):953-966, 2003.

[33] B.F. Feeny and R. Kappagantu. On the physical interpretation of proper orthogonal modes in vibrations. Journal of sound and vibration, 211(4):607616, 1998. 
[34] H. Leclerc, J. Neggers, F. Mathieu, S. Roux, and F. Hild. Correli 3.0. Agence pour la Protection des Programmes, Paris, 2015. IDDN.FR.001.520008.000.S.P.2015.000.31500.

[35] F. Mathieu, H. Leclerc, F. Hild, and S. Roux. Estimation of elastoplastic parameters via weighted femu and integrated-dic. Experimental Mechanics, 55(1):105-119, 2015.

[36] F. Hild, A. Bouterf, L. Chamoin, H. Leclerc, F. Mathieu, J. Neggers, F. Pled, Z. Tomičević, and S. Roux. Toward 4D Mechanical Correlation. Advanced Modeling and Simulation in Engineering Sciences, 3(1):17, 2016.

[37] J. Neggers, O. Allix, F. Hild, and S. Roux. Big data in experimental mechanics and model order reduction: Today's challenges and tomorrow's opportunities. Archives of Computational Methods in Engineering, Jul 2017. 